Hamam Abd* and Andreas König

\title{
A Compact Four Transistor CMOS-Design of a Floating Memristor for Adaptive Spiking Neural Networks and Corresponding Self-X Sensor Electronics to Industry 4.0
}

\author{
Ein kompaktes CMOS-Design aus vier Transistoren eines Floating Memristors für adaptive pulsende \\ neuronale Netzwerke und darauf aufbauende Self-X-Sensorelektronik für Industrie 4.0
}

DOI 10.1515/teme-2020-0024

\begin{abstract}
In this work we present, in the context of the transition from amplitude to robust spike domain sensing and electronics, a floating memristor. It can be used to construct memristor SNNs used for noise-robust conditioning and analog-to-digital conversion and manufactured using leading-edge technologies with more 'cranky' devices, low-voltage, low power, and minimal area on-chip. Also, this supports both machine learning as well as the self-x properties in advanced sensor electronics system for industry 4.0. The proposed memristor has less design complexity and a higher number of resistance levels as compared to other existing memristors. The proposed CMOS memristor is designed using AMS 0.35 $\mu \mathrm{m}$ CMOS technology and Cadence design tools. Its layout occupies an area of $70 \mu \mathrm{m} \times 85 \mu \mathrm{m}$. The simulation shows the performance of the proposed floating memristor emulator in the temperature range $\left(-40{ }^{\circ} \mathrm{C}\right.$ to $\left.85^{\circ} \mathrm{C}\right)$ and Monte-Carlo simulation.
\end{abstract}

Keywords: Frequency sensory, Memristor, Memristor neuromorphic architectures, Adaptive synapse, Adaptive spiking neural network, Self-x properties, Machine learning, Sensor electronics system, Industry 4.0.

Zusammenfassung: In dieser Arbeit stellen wir im Kontext des Übergangs von einer Amplituden-zur robusten Pulsbereichsrepräsentation und Sensorelektronik, eine Floating-Memristor-Schaltung vor. Diese soll als Synapsenelement der Erstellung von adaptiven pulsenden Neuronennetzen dienen, die Sensorsignalkonditionierung und -nach-Digitalwandlung robust gegenüber Herstellungs-

\footnotetext{
*Corresponding author: Hamam Abd, Lehrstuhl Integrierte Sensorsysteme (ISE), TU Kaiserslautern Deutschland, E-Mail: Abd@eit.uni-kl.de
}

Andreas König, Lehrstuhl Integrierte Sensorsysteme (ISE), TU Kaiserslautern Deutschland, E-Mail: koenig@eit.uni-kl.de schwankungen, sinkenden Betriebsspanunngen und reduziertem Aussteuerbereich, Verlustleistungs- und Flächenmimimierungsforderungen sowie Störeinflüßen erlauben und damit auch in modernsten Herstellungstechnologien mit Bauelementen sinkender Zuverlässigkeit zu lebensfähigen Sensorelektronik-Chips führen. Mit der dann gegebenen Adaptivität wird sowohl das Maschinelle Lernen unterstützt als auch die Forderung an Self-x-Eigenschaften der fortgeschrittenen Sensorelektronik für die Industrie 4.0 erfüllt. Der vorgeschlagene Memristor hat eine geringere Entwurfskomplexität und eine höhere Anzahl einstellbarer Widerstandsstufen im Vergleich zu anderen bestehenden Memristoren. Der CMOS-Memristor wurde in der AMS 0.35 um CMOS-Technologie mit der CadenceEntwurfsumgebung entworfen. Das zugehörige Layout belegt eine Fläche von $70 \mu \mathrm{m} \times 85 \mu \mathrm{m}$. Die Simulation bestätigt die Leistungsfähigkeit der vorgeschlagenen FloatingMemristor-Schaltung im Temperaturbereich $-40^{\circ} \mathrm{C}$ bis $85^{\circ} \mathrm{C}$ und einer Monte-Carlo-Analyse.'

Schlüsselwörter: Frequenzsensorik, Memristor, Neurmorphe Memristor-Architekturen, Adaptive Synapsen, Adaptives pulsendes neuronales Netzwerk, Self-x-Eigenschaften, Maschinelles Lernen, Sensorelektroniksystem, Industrie 4.0 .

\section{Introduction}

In the last few years, the number of edge devices in IoT and industry 4.0 has been increased leading to a growth in machine learning as well as the urgent need for self-x (self-healing, self-calibration) technology [1, 37, 38]. Issues of leading-edge technologies for integrated sensor electronics advocate the transition from amplitude to time or spike domain information processing. Inspirations from frequency sensors and adaptive spiking neural networks 
can be employed for resilient/self-x system realizations. A key mandatory element is a programmable or adaptive synapse, e.g. by a memristor realization. On the other hand, the memristor neuromorphic architectures learning, self-x, scalability, have a low power profile and small onchip footprint [20]. Currently, this technique became a promising solution for edge devices. Although many memristor neuromorphic architectures were proposed in the sensing system, however, the memristor SNNs (Spiking Neural Network) have a lower power profile and more robust to the noise since they encode the information using the spike times and process only when the events occur [31]. Also, from the mechanisms function view, since the SNNs use the time domain encoding (noise-robust) and have the capability of learning and self-x, they solve one of the main problems in the sensing system where the output noise of the sensor could lead to an indirect error in the measurements [31].

A lucid example of adaptive SNN are delay lines in acoustic localization, as shown in (Fig. 1) [14-16, 19]. The brain determines the location of the sound by the difference time of ears $=t_{1}-t_{2}$ where $t_{1}$ and $t_{2}$ are the times of the sound reaching the right and left ears, respectively as shown in (Fig. 1 A). The neural network in (Fig. 1 B) mimics the sound location detection by the brain. The network consists of two sensors, and each sensor represents one ear, two neurons, and two synapses. The first and the second neuron fire responds to the right and the left, respectively. The neuron spikes when the membrane voltage is greater than the threshold voltage, as shown in the (Fig. $1 \mathrm{~B}$ ). The synapses adaptively control the amount of the charge to the neuron by adapting the spike. Consequently, the synapses control the time delay at where the neurons will be spiked. The delay can be controlled by changing the weights of the synapses. These synapses are programmed with a certain weight to keep it constant under variations/perturbations. The synapses can be emulated by a memristor with one transistor (1T1M).

A memristor is a two-terminal device where the resistance value of the memristor is dependent on the polarity and magnitude of the applied voltage and the length of time that voltage has been applied [8]. As reviewed in the literature, the memristor concept was suggested by Leon Chua in 1971[8]. However, researches could not evaluate the memristor until it was implemented by Hewlett Packard (HP) in 2008 [29]. Even though many benefits gained from using the memristors in much application such as neuromorphic circuits[20], programmable analog circuits[24], chaotic circuits[23], adaptive filters[9], basic arithmetic operations[18], sensory electronics system, internet of things (IoT), machine learning[12], they are not

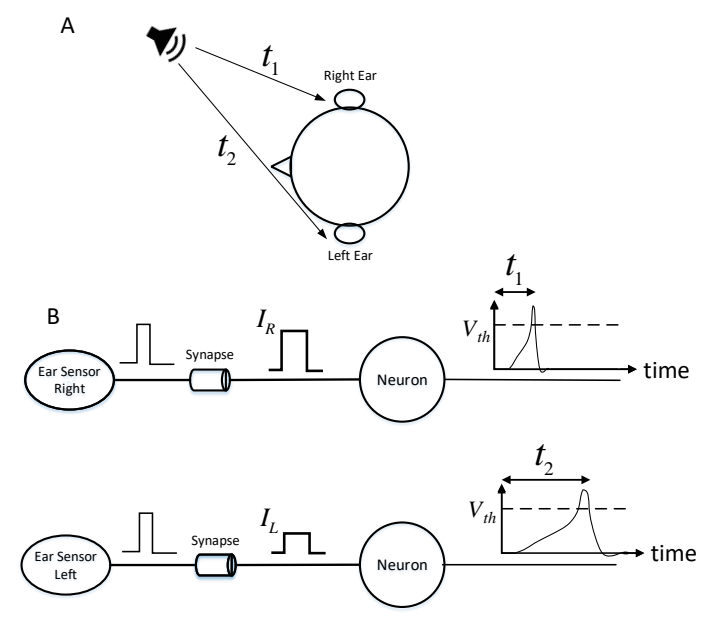

Fig. 1: (A) Acoustic localization model. (B) SNN schematic structure of equivalent conversion concept.

expected to be used in the commercial chip soon because of many open problems that need to be investigated, such as the fabrication complexity of memristor systems, unstable switching behavior, the finite number of resistor levels, and compatibility with CMOS technology [20]. To implement neuromorphic architectures and integrated on the real chips, therefore, the researchers have focused on memristor emulators [2-6, 10, 17, 27, 28, 30, 32-36]. State-of-theart designs cannot integrate on-chip memristor because they have complex circuits and need an external devices. They are used a lot of active components and passive elements $[2-6,10,17,27,28,30,32-36]$. Some of them have grounded structures $[2,6,10,17,32,34,35]$, and others have floating structures [3-5, 27, 28, 30, 33, 36]. Designing a floating memristor circuit is more complex than designing a grounded memristor circuit; however, grounded memristor has limited applications. In [17], authors proposed grounded memristor with two operational amplifiers, ten transistors, two resistors, a capacitor and multiplier. Also, in [2], they proposed grounded memristor emulator circuit using current backward transconductance amplifier (CBTA), multiplier, two resistors and a single capacitor. Additionally, in [6], they proposed grounded memristor emulator with four transistors. Likewise, in [10], he proposed grounded memristor emulator with four transistors. Similarly, in [32] he proposed grounded memristor emulator with seven transistors and a single capacitor. Moreover, in [35] they proposed grounded memristor emulator circuit using voltage differencing current conveyor (VDCC), two transistors and a single capacitor. As well, in [34] they proposed grounded memristor emulator circuit using voltage difference transconductance amplifier (VDTA), single 
transistors. In [28], floating memristor emulator circuit was proposed using three operational transconductance amplifiers (OTA), four second-generation current conveyors (CCII), six resistors and a single capacitor. Also, in [5] floating memristor was proposed with MO-OTA, resistor, capacitor, and multiplier. Besides, in [30] floating memristor was proposed with three transistors, external capacitor and a current source. Also, in [36] they proposed a floating memristor emulator circuit using current backward transconductance amplifier (CBTA) with two capacitors. Likewise, in [4] they proposed floating memristor with single OTA, two transistors and an external capacitor. Moreover, in [3] they proposed floating memristor with ten transistors and three external capacitors. Additionally, in [33] they proposed floating memristor emulator circuit using differential difference current conveyor (DDCC) two resistors, capacitor and multiplier. Furthermore, in [27] they proposed a floating memristor emulator circuit, which is built with four second-generation current conveyors (CCII) and single multiplier.

The primary objective of this research is to design a memristor that can be on-chip integrated without needing any external components. The target is to construct an adaptive synapse and an adaptive spiking neuron. These will serve to construct memristor-based adaptive SNNs, that introduce machine learning to support sensor electronics with self-x properties as demanded by industry 4.0 .

\section{Proposed Methodology}

In this research, we design a compact floating memristor emulator circuit consisting of only four CMOS transistors, as shown in (Fig. 2). The proposed memristor has the following distinguishing features as compared to the other work presented in $[2-6,10,17,27,28,30,32-36]: 1)$ less complexity. 2) can be on a chip integrated. 3) does not need any external device. 4) has a high number of resistor levels. The drain-to-source resistance of MOSFET (M1) represents the resistance of the memristor, which is changing by the voltage across the transistor M4. M4 is conecting as a capacitor by tying the drain, source, and bulk to $V_{s s}$ [26]. M2 and M3 transistors are the feedback circuit that controls the voltage across M4. The feedback circuit is controlled by external voltage sources (VA, VB).

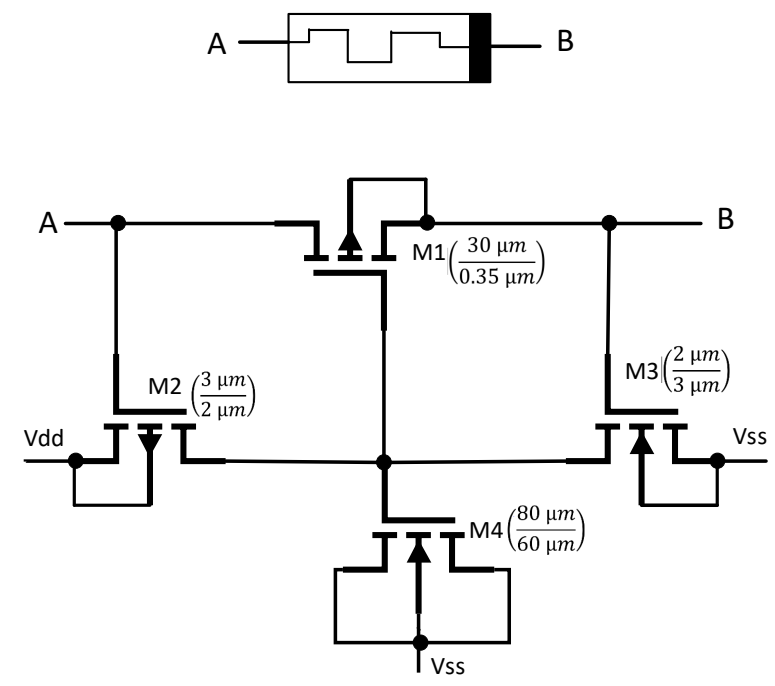

Fig. 2: Proposed emulator memristor circuit and its symbol.

\section{Results}

The proposed memristor is simulated using spectre simulator from Cadence. AMS 0.35 $\mu \mathrm{m}$ CMOS technology is used, and the power supply voltages of the circuit are $V_{d d}=-V_{s s}=1.65 \mathrm{~V}$. The aspect ratios of the transistors are designed as $(W / L)_{M 1}=30 \mu \mathrm{m} / 0.35 \mu \mathrm{m},(W / L)_{M 2}=$ $3 \mu \mathrm{m} / 2 \mu \mathrm{m},(W / L)_{M 3}=2 \mu \mathrm{m} / 3 \mu \mathrm{m}, \operatorname{and}(W / L)_{M 4}=$ $80 \mu \mathrm{m} / 60 \mu \mathrm{m}$. In order to draw the voltage-current characteristic of the proposed floating memristor, the sinusoidal signal has been applied to it, as shown in (Fig. 3).

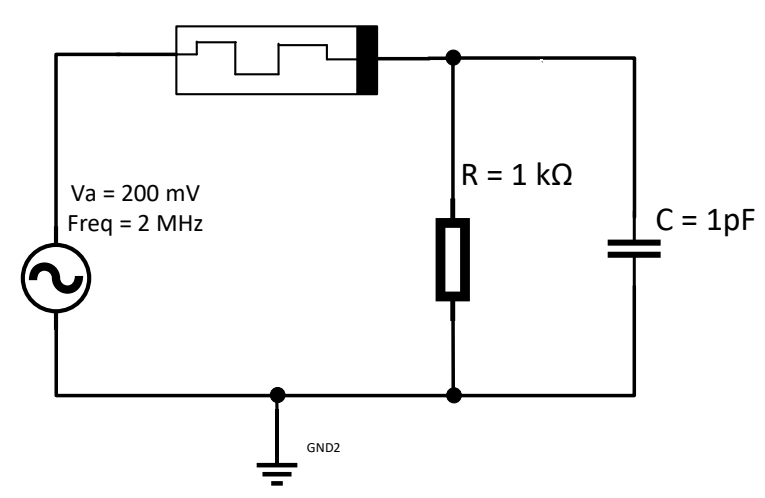

Fig. 3: The test circuit of the memristor characteristics.

The voltage-current characteristic of the proposed floating memristor for $2 \mathrm{MHz}$ frequency is shown in (Fig. 4). 


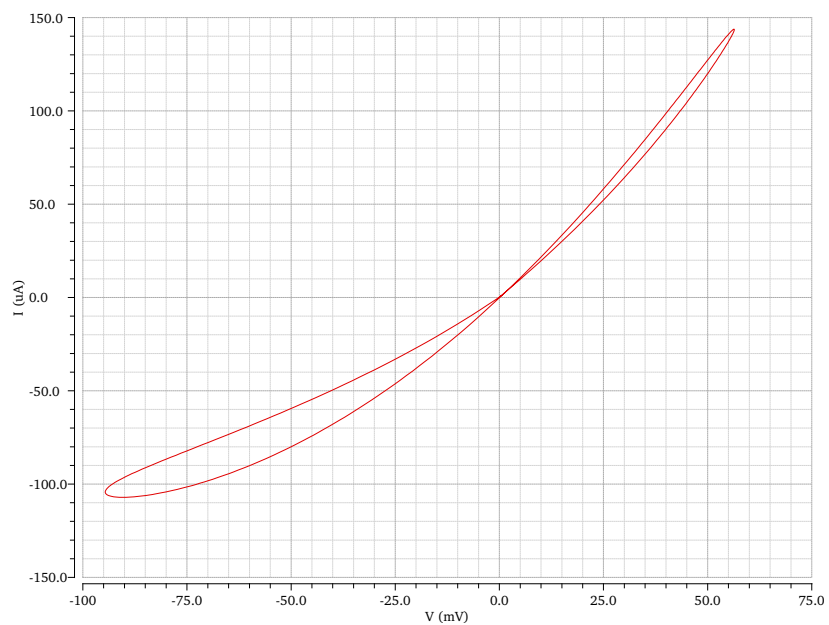

Fig. 4: The voltage-current characteristics of memristor for 2 $\mathrm{MHz}$.

The comparison of state of the art and this work are listed in (Tab. 1). The active and passive devices are recoded, operation frequency, emulator properties (floating or grounded) are listed in (Tab. 1).

The proposed memristor can achieve up to 783 resistance levels with adaptation time equal to $500 \mathrm{~ns}$ between each level. The results show that the proposed memristor can be programmed up to 9.61-bit values which are higher than the recent memristors proposed in $[7,11,13,21,22,25]$ where authors programmed up to $121,200,64,100,30$ and 24 levels, respectively. From the voltage-current characteristics of the memristor shown in (Fig. 4). Just like a biological synapse, the proposed memristor recovers the original state when the applied voltage is removed. Furthermore, the proposed memristors can be used as the weights of the synapses as well as to build the neuron for the memristor SNN as spiking adaptive sensor circuits. Moreover, the neurons built using this memristor do not need a reset after firing like the biological neurons. The proposed memristor with one transistor (1T1M) mimics synapse which can be programmed to 783 resistance levels using adapted pulses of $100 \mathrm{~ns}$ and controlling the transistor voltage gate to give more control of the memristor current as shown in (Fig. 5).

Monte-Carlo simulation of the $1 \mathrm{~T} 1 \mathrm{M}$ under temperature range $\left(-40{ }^{\circ} \mathrm{C}\right.$ to $\left.85^{\circ} \mathrm{C}\right)$ is given in (Fig. 6) to confirm workableness. The worst-case result is picked, as shown in (Fig. 7). In order to reset it the original weight of the synapse, the voltage of the gate transistor is adjusted, and the recovered result is shown in (Fig. 8).

The layout of the proposed floating memristor circuit (Fig. 9) in the figure which occupies $70 \mu \mathrm{m} * 85 \mu \mathrm{m}$ chip area.

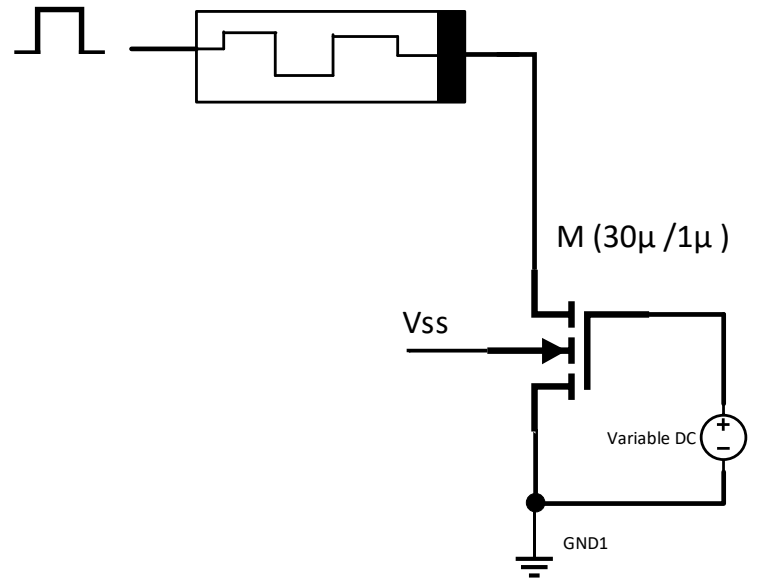

Fig. 5: Memristor with transistor (1T1M) architecture used to program the memristor.

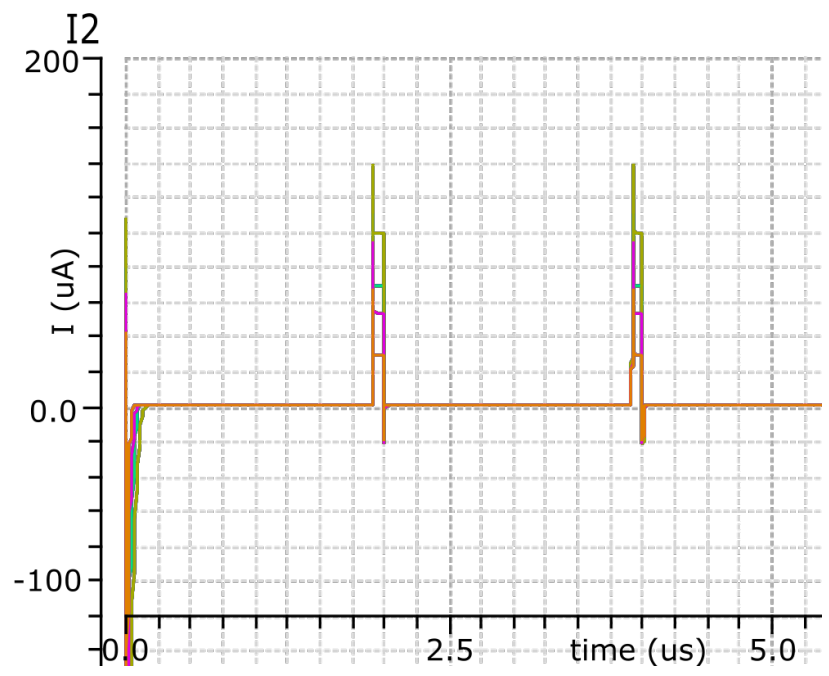

Fig. 6: Monte-Carlo simulation of the $1 \mathrm{~T} 1 \mathrm{M}$ in the temperature range $\left(-40^{\circ} \mathrm{C}\right.$ to $\left.85^{\circ} \mathrm{C}\right)$.

\section{Conclusions}

In this work, a compact floating memristor circuit is presented, which consists of only four MOS-transistors without requiring any external devices. By comparing it with the previous designs that needed active components like a multiplier, OP-AMP, OTA, CBTA, VDCC, VDTA, DDCC, CCII, and passive elements like capacitor and resistor. The proposed floating memristor is implemented using AMS 0.35 $\mu \mathrm{m}$ CMOS technology and simulated with cadence design tools in the temperature range $\left(-40{ }^{\circ} \mathrm{C}\right.$ to $85^{\circ} \mathrm{C}$ ) and Monte-Carlo model. The proposed floating memristor is suitable for an adaptive synapse and in an adaptive spiking neuron. 
Table 1: Comparison of the proposed memristor circuit with previous works.

\begin{tabular}{ccccc}
\hline Reference & Active component & Passive device & Frequency & Memristors type \\
\hline$[17]$ & 2 OP-AMPs, 1 Multiplier, 10 Transistors & $2 \mathrm{R}, 1 \mathrm{C}$ & $800 \mathrm{~Hz}$ & Grounded Memristor \\
{$[2]$} & CBTA, 1 Multiplier & $2 \mathrm{R}, 1 \mathrm{C}$ & $100 \mathrm{kHz}$ & Grounded Memristor \\
{$[6]$} & 4 transistors & - & $100 \mathrm{MHz}$ & Grounded Memristor \\
{$[10]$} & 4 transistors & - & $100 \mathrm{kHz}$ & Grounded Memristor \\
{$[32]$} & 7 transistors & $1 \mathrm{C}$ & $50 \mathrm{MHz}$ & Grounded Memristor \\
{$[35]$} & VDCC, 2 transistors & $1 \mathrm{C}$ & $2 \mathrm{MHz}$ & Grounded Memristor \\
{$[34]$} & VDTA, single transistors & - & $50 \mathrm{MHz}$ & Grounded Memristor \\
\hline$[28]$ & 3 OTA, 4 CCII & $6 \mathrm{R}, 1 \mathrm{C}$ & $10 \mathrm{kHz}$ & Floating Memristor \\
{$[5]$} & $1 \mathrm{MO}-$ OTA, 1 Multiplier & $1 \mathrm{R}, 1 \mathrm{C}$ & $1 \mathrm{kHz}$ & Floating Memristor \\
{$[30]$} & 3 transistors & $1 \mathrm{C}$ & $1 \mathrm{MHz}$ & Floating Memristor \\
{$[36]$} & $1 \mathrm{CBTA}$ & $2 \mathrm{C}$ & $1 \mathrm{MHz}$ & Floating Memristor \\
{$[4]$} & $1 \mathrm{OTA}, 2$ transistors & $1 \mathrm{C}$ & $30 \mathrm{~Hz}$ & Floating Memristor \\
{$[3]$} & 10 transistors & $3 \mathrm{C}$ & $5 \mathrm{~Hz}$ & Floating Memristor \\
{$[33]$} & $1 \mathrm{DDCC}, 1$ Multiplier & $2 \mathrm{R}, 1 \mathrm{C}$ & $1 \mathrm{MHz}$ & Floating Memristor \\
{$[27]$} & $4 \mathrm{CCII}, 1$ Multiplier & $5 \mathrm{R}, 1 \mathrm{C}$ & $20.2 \mathrm{kHz}$ & Floating Memristor \\
\hline
\end{tabular}

I2

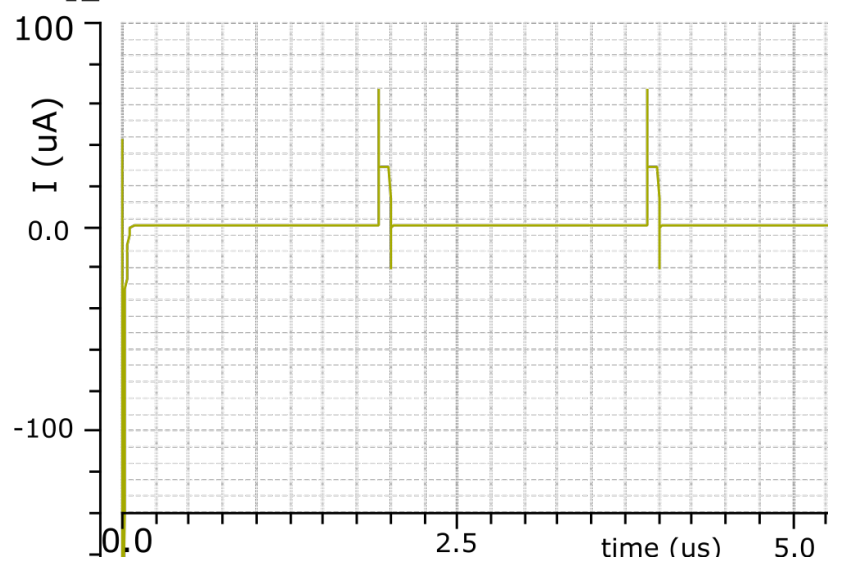

Fig. 7: Worst-case of the Monte-Carlo simulation for the $1 \mathrm{~T} 1 \mathrm{M}$.

\section{References}

[1] S. Alraho and A. König. Wide input range, fully-differential indirect current feedback instrumentation amplifier for self$x$ sensory systems/symmetrischer instrumentierungsverstärker mit indirekter stromgegenkopplung und hoher eingangsignalspanne für integrierte sensorsysteme mit self-xeigenschaften. tm-Technisches Messen, 86(s1):62-66, 2019.

[2] U. E. Ayten, S. Minaei, and M. Sağbaș. Memristor emulator circuits using single cbta. AEU-IJEC, 82:109-118, 2017.

[3] Y. Babacan and F. Kaçar. Memristor emulator with spiketiming-dependent-plasticity. AEU-IJEC, 73:16-22, 2017.

[4] Y. Babacan and F. Kaçar. Floating memristor emulator with subthreshold region. AICSP, 90(2):471-475, 2017.

[5] Y. Babacan, A. Yesil, and F. Kacar. Memristor emulator with tunable characteristic and its experimental results. AEU-IJEC, 81:99-104, 2017.

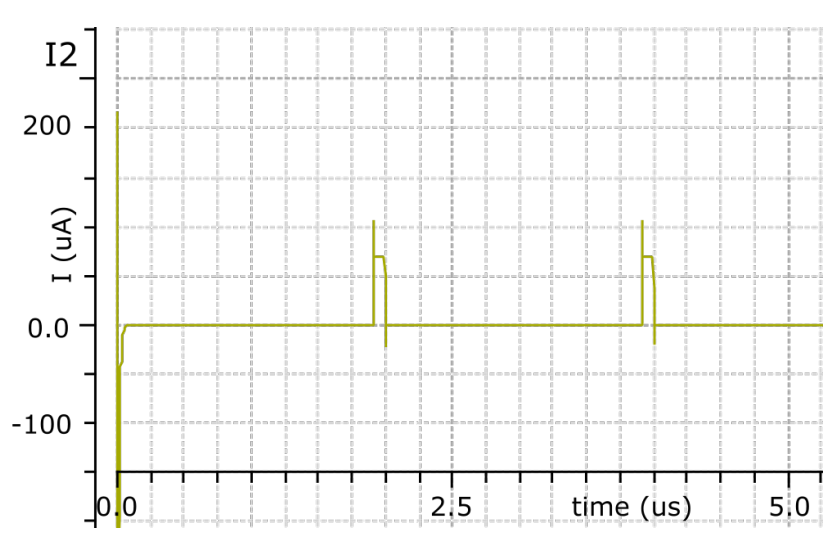

Fig. 8: Recovered the worst-case of the Monte-Carlo simulation for the $1 \mathrm{~T} 1 \mathrm{M}$.

[6] Y. Babacan, A. Yesil, and F. Gul. The fabrication and mosfetonly circuit implementation of semiconductor memristor. IEEE TED, 65(4):1625-1632, 2018.

[7] J. Chen, W.-Q. Pan, Y. Li, R. Kuang, Y.-H. He, C.-Y. Lin, N. Duan, G.-R. Feng, H.-X. Zheng, T.-C. Chang, et al. Highprecision symmetric weight update of memristor by gate voltage ramping method for convolutional neural network accelerator. IEEE EDL, 41(3):353-356, 2020.

[8] L. Chua. Memristor-the missing circuit element. IEEE Transactions on circuit theory, 18(5):507-519, 1971.

[9] T. Driscoll, J. Quinn, S. Klein, H.-T. Kim, B. Kim, Y. V. Pershin, M. Di Ventra, and D. Basov. Memristive adaptive filters. APL, 97(9):093502, 2010.

[10] F. Gul. Circuit implementation of nano-scale tio 2 memristor using only metal-oxide-semiconductor transistors. IEEE EDL, 40(4):643-646, 2019.

[11] I. Gupta, A. Serb, A. Khiat, R. Zeitler, S. Vassanelli, and T. Prodromakis. Real-time encoding and compression of neuronal spikes by metal-oxide memristors. Nature communications, 7(1):1-9, 2016. 


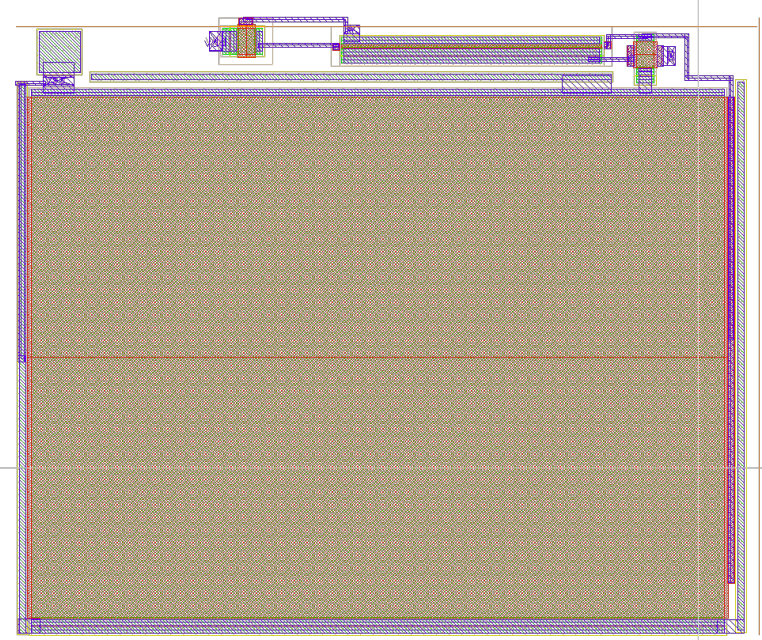

Fig. 9: The layout of the proposed floating memristor emulator circuit.

[12] M. Gusev and S. Dustdar. Going back to the roots-the evolution of edge computing, an iot perspective. IEEE IC, 22 (2):5-15, 2018.

[13] H. Jiang, L. Han, P. Lin, Z. Wang, M. H. Jang, Q. Wu, M. Barnell, J. J. Yang, H. L. Xin, and Q. Xia. Sub-10 nm ta channel responsible for superior performance of a hfo 2 memristor. Scientific reports, 6:28525, 2016.

[14] A. C. Kammara and A. Koenig. Contributions to integrated adaptive spike coded sensor signal conditioning and digital conversion in neural architecture. In Sensors and Measuring Systems 2014; 17. ITG/GMA Symposium, pages 1-6. VDE, 2014.

[15] A. C. Kammara and A. König. Increasing the resolution of an integrated adaptive spike coded sensor to digital conversion neuro-circuit by an enhanced place coding layer. In Symposium des AHMT, volume 9, page 2014, 2014.

[16] A. Kammara S., Abhaya Chandra König. Robust adcs for dependable integrated measurement systems based on adaptive neuromorphic spiking realization. XXIX Messtechnisches Symposium, pages 157-164, 2015.

[17] H. Kim, M. P. Sah, C. Yang, S. Cho, and L. O. Chua. Memristor emulator for memristor circuit applications. IEEE TCS, 59(10):2422-2431, 2012.

[18] V. P. S. Kirar. Memristor: the missing circuit element and its application. International Journal of Electrical, Computer, Energetic, Electronic and Communication Engineering, 6: 1395-1397, 2012.

[19] A. König et al. Ssdc $\alpha$-inherently robust integrated biomimetic sensor-to-spike-to-digital converter based on peripheral neural ensembles. tm-Technisches Messen, 83(9): 531-542, 2016.

[20] O. Krestinskaya, A. P. James, and L. O. Chua. Neuromemristive circuits for edge computing: A review. IEEE TNNLS, 31 (1):4-23, 2020.

[21] C. Li, D. Belkin, Y. Li, P. Yan, M. Hu, N. Ge, H. Jiang, E. Montgomery, P. Lin, Z. Wang, et al. Efficient and self- adaptive in-situ learning in multilayer memristor neural networks. Nature communications, 9(1):1-8, 2018.

[22] E. J. Merced-Grafals, N. Dávila, N. Ge, R. S. Williams, and J. P. Strachan. Repeatable, accurate, and high speed multi-level programming of memristor $1 \mathrm{t} 1 \mathrm{r}$ arrays for power efficient analog computing applications. Nanotechnology, 27 (36):365202, 2016.

[23] B. Muthuswamy. Implementing memristor based chaotic circuits. IJBC, 20(05):1335-1350, 2010.

[24] Y. V. Pershin and M. Di Ventra. Practical approach to programmable analog circuits with memristors. IEEE TCSI, 57(8):1857-1864, 2010.

[25] K. V. Pham, S. B. Tran, T. V. Nguyen, and K.-S. Min. Asymmetrical training scheme of binary-memristor-crossbarbased neural networks for energy-efficient edge-computing nanoscale systems. Micromachines, 10(2):141, 2019.

[26] B. Razavi. Design of analog CMOS integrated circuits. Tata McGraw-Hill Education, 2017.

[27] C. Sánchez-López, J. Mendoza-Lopez, M. Carrasco-Aguilar, and C. Muñiz-Montero. A floating analog memristor emulator circuit. IEEE Transactions on Circuits and Systems II: Express Briefs, 61(5):309-313, 2014.

[28] H. Sözen and U. Çam. Electronically tunable memristor emulator circuit. AICSP, 89(3):655-663, 2016.

[29] D. B. Strukov, G. S. Snider, D. R. Stewart, and R. S. Williams. The missing memristor found. nature, 453(7191): 80-83, 2008.

[30] J. Vista and A. Ranjan. A simple floating mos-memristor for high-frequency applications. IEEE TVLSIS, 27(5):1186-1195, 2019.

[31] Q. Xia and J. J. Yang. Memristive crossbar arrays for braininspired computing. Nature materials, 18(4):309-323, 2019.

[32] A. Yesil. A new grounded memristor emulator based on mosfet-c. AEU-International Journal of Electronics and Communications, 91:143-149, 2018.

[33] A. Yeșil, Y. Babacan, and F. Kaçar. A new ddcc based memristor emulator circuit and its applications. MJ, 45(3): 282-287, 2014.

[34] A. Yeșil, Y. Babacan, and F. Kaçar. Design and experimental evolution of memristor with only one vdta and one capacitor. IEEE Transactions on Computer-Aided Design of Integrated Circuits and Systems, 38(6):1123-1132, 2018.

[35] A. Yesil, Y. Babacan, and F. Kacar. Electronically tunable memristor based on vdcc. AEU-International Journal of Electronics and Communications, 107:282-290, 2019.

[36] A. Yesil, Y. Babacan, and F. Kacar. A new floating memristor based on cbta with grounded capacitors. JCSC, page 1950217, 2019.

[37] Q. Zaman and A. König. Self-x integrated sensor circuits immune to measurement noise in the presence of input perturbation by using robust optimization/integrierte sensorelektronik mit self-x-eigenschaften und verbesserter immunität gegenüber rauscheinflüssen bei der messung und störung der eingangsgrößen durch ein robustes optimierungsverfahren. tm-Technisches Messen, 86(s1):107-111, 2019.

[38] Q. Zaman, S. Alraho, and A. König. Robust optimization of self-x sensory electronics in presences of environmental variations for industry 4 . 0. Sensor and Measurement Science International, pages 295-296, 2020. 